\title{
Choroidal Metastasis of Adenocarcinoma of the Lung Presenting as Pigmented Choroidal Tumor
}

\author{
Shahar Frenkel Jacob Pe'er \\ Department of Ophthalmology, Hadassah-Hebrew University Medical Center, \\ Jerusalem, Israel
}

\section{Key Words}

Choroidal metastasis · Pulmonary adenocarcinoma · Pigmented choroidal tumor

\begin{abstract}
Our case represents a unique occurrence of pigmented choroidal tumor that clinically appeared as choroidal melanoma and was treated accordingly. At the same time, while evaluating the patient for systemic metastases of uveal melanoma, she was diagnosed as having lung carcinoma. Events that led to the enucleation of the eye enabled a histological diagnosis: pulmonary adenocarcinoma. In our case, it happened that a very rare pigmented choroidal metastatic tumor was the presenting sign of a pulmonary adenocarcinoma.
\end{abstract}

\section{Case Report}

An 84-year-old woman was referred to our ocular oncology service with a suspected diagnosis of choroidal melanoma in her left eye. Upon examination in our clinic, the visual acuity in her right eye was 0.7 and in the left eye, hand movements. The right eye showed progressive cataract and normal fundus. The left eye was pseudophakic, and an elevated pigmented tumor with overlying hemorrhage was seen in the nasal side of the fundus, with hemorrhagic retinal detachment surrounding it (fig, 1 ).

During ultrasound examination, a dome-shaped tumor was seen at 9 o'clock anterior to the equator. The basal diameter measured $13.4 \times 12.2 \mathrm{~mm}$, and the maximal height was $6.9 \mathrm{~mm}$. The internal reflectivity was medium-low, although irregular. Retinal detachment surrounded the tumor, with blood under the retina (fig. 2).

Based on the clinical appearance and ultrasound (not typical, but suggestive), the diagnosis of left eye choroidal melanoma was made. The tumor was treated using Ru-106 brachytherapy, irradiating 100,000 cGy to the tumor base. The response to the treatment was very good, with decrease of the tumor maximal height to $4.0 \mathrm{~mm}$ ( $\underline{\text { fig. } 3}$ ). 
During evaluation for systemic metastases, a space-occupying lesion was seen by CT in the right upper lobe of the lung along with many nodules in other parts of the lung that were interpreted as metastatic spread. The liver and the rest of the abdominal imaging were normal. The findings were also confirmed by PET-CT. A 'hot' lesion was also seen in the right lobe of the thyroid. The patient underwent biopsy of the tumor in the lung, with a suspicion of metastatic uveal melanoma. However, the biopsy showed well-differentiated adenocarcinoma of the lung. The lung malignancy was treated in another hospital by chemotherapy and radiation therapy.

During the first year of follow-up, the remnant of the tumor remained stable. Fourteen months after treatment, regrowth was noticed, which increased upon examination 3 months later (fig. 4). Because maximal irradiation had already been applied, enucleation was performed and the eyeball was sent to our ophthalmic pathology laboratory for evaluation.

Microscopic examination revealed an eye with a tumor mass occupying a portion of the choroid from the pars plana up to close to the $\mathrm{ONH}$. The tumor was composed of sheets of cuboidal ciliated cells arranged around spaces in a papillary formation ( $\underline{\text { fig. } 5}$ ). Immunostaining was positive for cytokeratin (CK) and thyroid transcription factor-1 (TTF-1) (ig. 6), and negative for MART-1, S-100 and thyroglobulin. No extraocular extension was noted. The findings were compatible with pulmonary papillary adenocarcinoma metastasis to the choroid.

\section{Discussion}

Metastatic tumors of the uvea are considered to be the most common form of intraocular malignancy in adults [1]. The prevalence of metastases to the ocular region in patients with known disseminated cancer ranges from as low as $0.07 \%$ to as high as $27 \%[2,3]$. The possible increase in incidence in the last decades may reflect a longer survival of cancer patients. It may also be the result of heightened awareness of the treating physician, enabling a more prompt and accurate diagnosis of metastatic spread to the choroid. The incidence of choroidal metastases may be even higher, considering that many cases are asymptomatic or may remain undiagnosed due to the critical general condition of many of these patients who do not come to the attention of an ophthalmologist.

The vast majority of the uveal metastatic tumors are from carcinomas. Metastatic cutaneous melanoma to the eye is rare. Metastases of sarcomas to the eye are very rare. The most common primary tumor metastasizing to the uvea is breast carcinoma, while the second most common is lung carcinoma $[4,5]$. Choroidal metastases may be the presenting sign of systemic malignancy, and this is mainly true for metastatic lung carcinoma $[4,5]$. This highlights the vital role that ophthalmologists play in bringing such patients to medical attention.

Uveal metastatic tumors are usually amelanotic, except for metastases from cutaneous melanoma, which can be pigmented. Pigmented metastases of nonmelanotic tumors to the uvea are extremely rare, and a search of the literature revealed only 1 publication with such a metastatic tumor: a choroidal metastasis of a pigmented neuroendocrine tumor [6]. In that case, as in ours, the initial clinical diagnosis was choroidal melanoma, and our patient was treated accordingly, using brachytherapy. If the patient had not had regrowth that led to enucleation and histological and immunohistochemical diagnoses, we would probably have stayed with the incorrect diagnosis of choroidal melanoma. In some choroidal metastatic lesions, pigment mottling is seen over the surface of the tumor [7, 8], but the tumor itself does not appear pigmented, as in our case. 
Uveal melanoma is often diagnosed in patients with other malignancies, usually carcinomas [9]. Therefore, the finding of lung carcinoma in a patient with a presumed diagnosis of choroidal melanoma was not unexpected, especially when the clinical appearance of the pigmented choroidal tumor was very suggestive of choroidal melanoma.

Serous detachment is a common finding in eyes with choroidal metastatic tumor [1]. However, hemorrhagic detachment in such cases is rare, and only 1 such report could be found in the literature [10]. It is more frequent in eyes with uveal melanoma. In our case, however, this subretinal hemorrhage was absorbed after the brachytherapy and tumor response, and only some remnants in the form of a cholesterol cleft could be found in the histological evaluation.

The histopathological diagnosis of choroidal metastasis of papillary adenocarcinoma in our patient was quite surprising. In order to investigate the primary tumor, some immunohistochemical staining was used. The positive CK and negative S-100 and MART-1 confirmed the diagnosis of epithelial tumor, and ruled out the diagnosis of melanoma. TTF-1 is a tissue-specific transcription factor expressed in the epithelial cells of the thyroid and lung, and carcinomas arising from the lung and thyroid also show frequent TTF-1 expression [11,12]. Among lung carcinomas, TTF-1 is expressed specifically in pulmonary adenocarcinoma. In our case, the staining for thyroglobulin was negative, indicating lung carcinoma origin for the choroidal metastasis, and not thyroid carcinoma origin, as could be suspected because of the 'hot' lesion found in the thyroid.

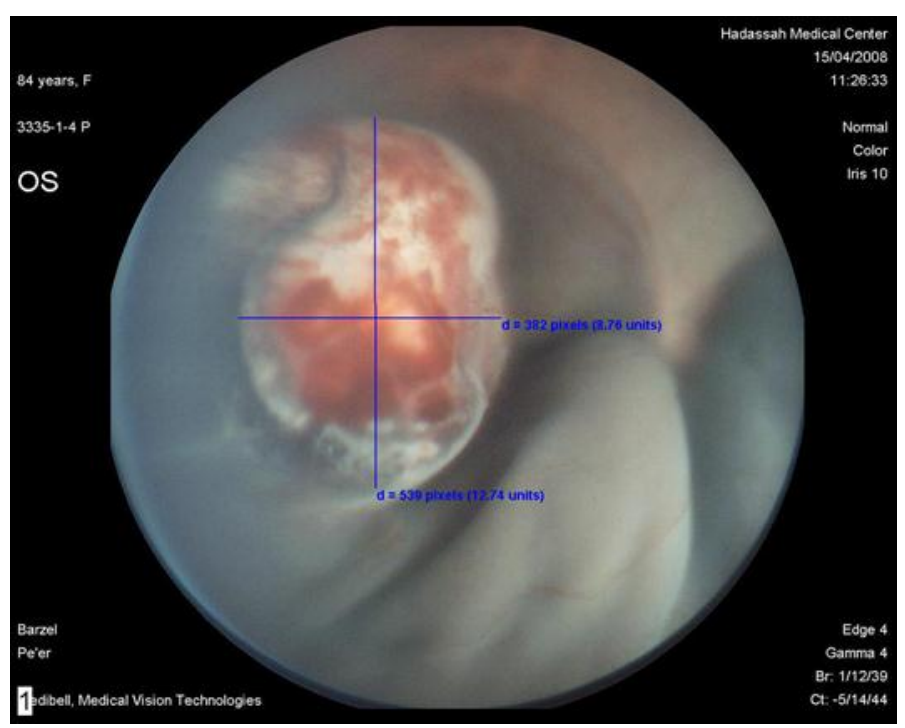

Fig. 1. Left eye fundus picture shows an elevated pigmented tumor with hemorrhage above and hemorrhagic retinal detachment surrounding the tumor. 


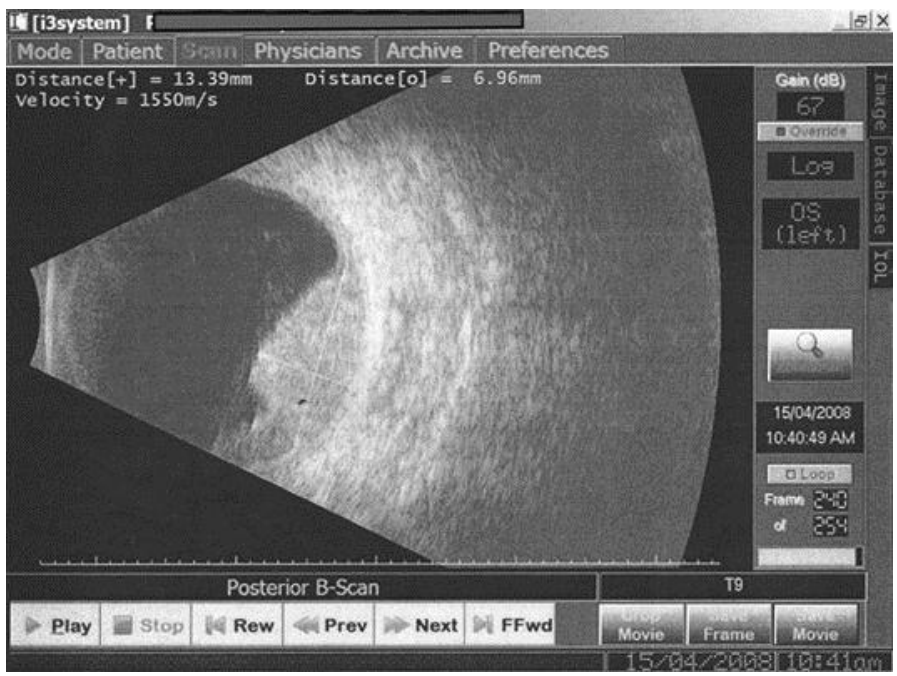

Fig. 2. B-scan ultrasonography shows an elevated mass in the nasal side of the globe anterior to the equator.

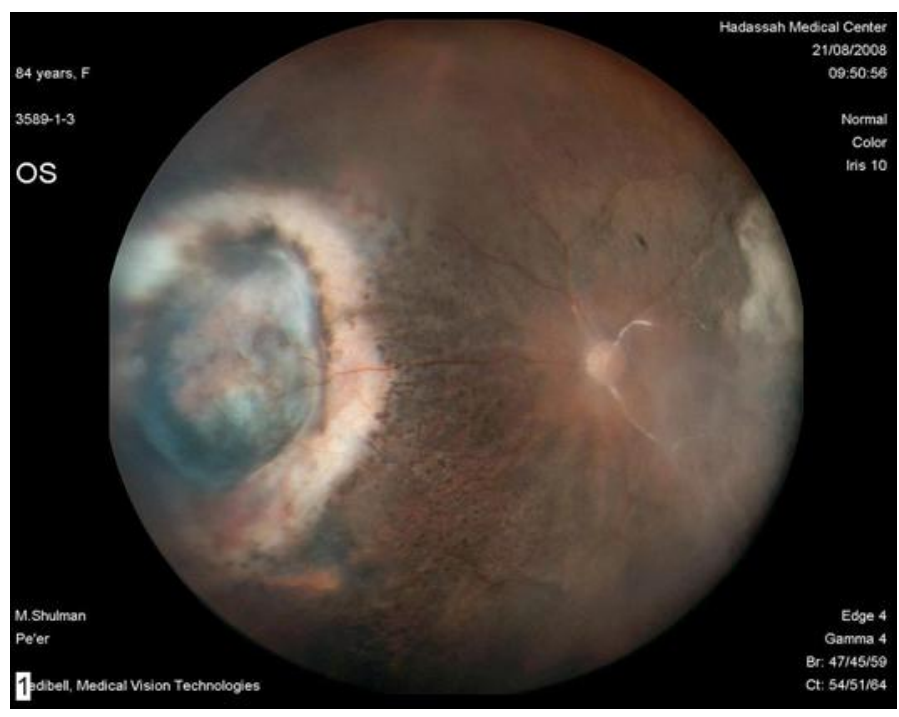

Fig. 3. Left eye fundus picture with the pigmented tumor, after being treated by brachytherapy, surrounded by atrophic scar tissue. 


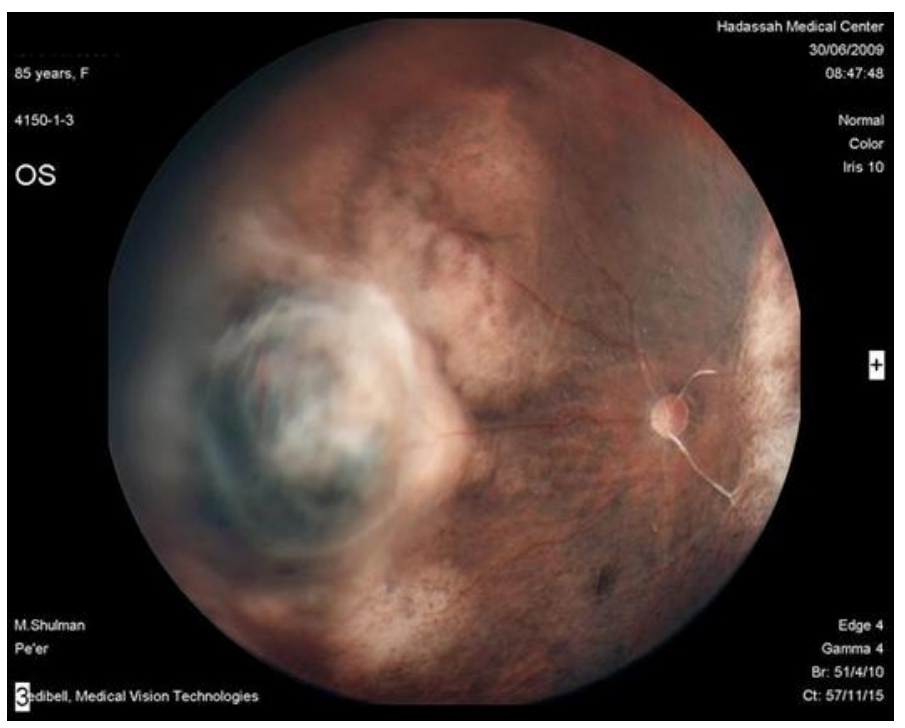

Fig. 4. Left eye fundus picture shows regrowth of the pigmented tumor.

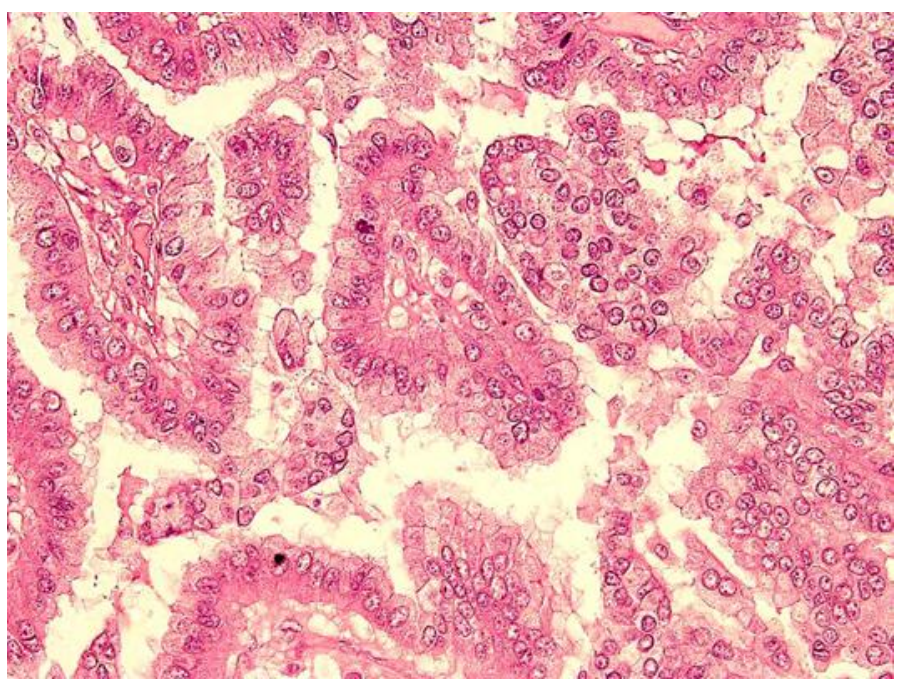

Fig. 5. Histological picture of the tumor in the enucleated eye shows a lesion composed of sheets of cuboidal ciliated cells arranged around a central core in a papillary formation (H\&E. $\times 200$ ). 


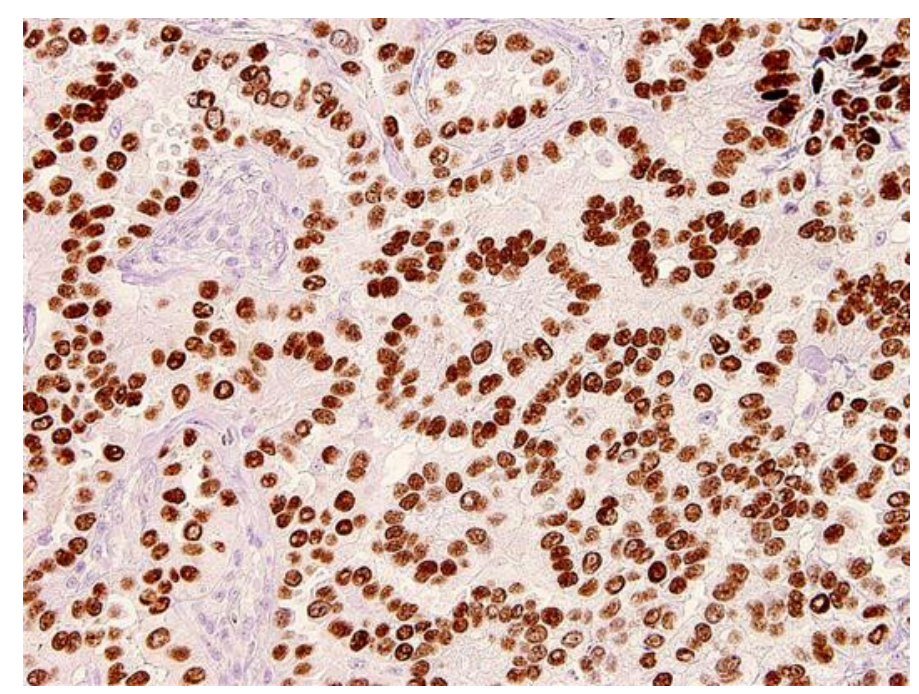

Fig. 6. Histological picture of the tumor in the enucleated eye shows positive TTF- 1 immunostaining $(\times 200)$.

\section{References}

1 Shields CL, Shields JA, Gross NE, Schwartz GP, Lally SE: Survey of 520 eyes with uveal metastases. Ophthalmology 1997;104:1265-1276.

$\checkmark 2$ Godtfredsen E: On the frequency of secondary carcinoma in the choroid. Acta Ophthalmologica 1944;22:394-400.

-3 Bullock JD, Yanes B: Ophthalmic manifestations of metastatic breast cancer. Ophthalmology 1980;87:961-973.

4 Shields JA, Shields CL: Metastatic tumors to the intraocular structures; in Shields JA, Shields CL (eds): Intraocular Tumors: A Text and Atlas. Philadelphia, Saunders, 1992, pp 7-237.

5 Amer R, Pe'er J, Chowers I, Anteby I: Treatment options in the management of choroidal metastases. Ophthalmologica 2004;218:372-377.

6 Eagle RC Jr, Ehya H, Shields JA, Shields CL: Choroidal metastasis as the initial manifestation of a pigmented neuroendocrine tumor. Arch Ophthalmol 2000;118:841-845.

7 Natesh S, Chin KJ, Finger PT: Choroidal metastases fundus autofluorescence imaging: correlation to clinical, OCT, and fluorescein angiographic findings. Ophthalmic Surg Lasers Imaging 2010;41:406-412.

8 Meziani L, Cassoux N, Le Rouic LL, et al: Uveal metastasis revealing lung cancer. J Fr Ophthalmol 2012;35:420-425.

$\checkmark 9$ Diener-West M, Reynolds SM, Aguglilaro DJ, et al: Collaborative Ocular Melanoma Study Group: Second primary cancers after enrollment in the COMS trials for treatment of choroidal melanoma: COMS Report NO. 25. Arch Ophthalmol 2005;123:601-604.

10 Dugan JD Jr, Repke C, Wong T, Eagle RC Jr, Tasman W: Massive spontaneous subretinal hemorrhage from a choroidal metastasis. Am J Ophthalmol 1993;115:677-679.

11 Katoh R, Kawaoi A, Miyagi E, et al: Thyroid transcription factor-1 in normal, hyperplastic and neoplastic follicular thyroid cells examined by immunohistochemistry and nonradioactive in-situ hybridization. Mod Pathol 2000;13:570-576.

12 Stenhouse G, Fyfe N, King G, et al: Thyroid transcription factor 1 in pulmonary adenocarcinoma. J Clin Pathol 2004;57:383-387. 